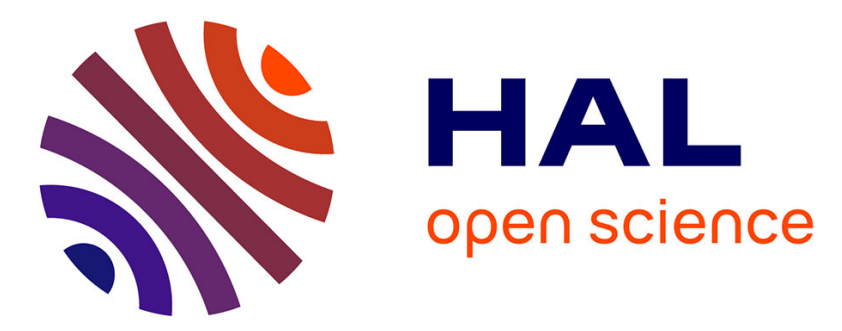

\title{
Optimisation de la résistance au cisaillement interlaminaire (RCIL) des matériaux composites fabriqués par LRI en fonction des paramètres de fabrication
}

\author{
Alvine Njionhou Kepnang, Florentin Berthet, Bruno Castanié
}

\section{To cite this version:}

Alvine Njionhou Kepnang, Florentin Berthet, Bruno Castanié. Optimisation de la résistance au cisaillement interlaminaire (RCIL) des matériaux composites fabriqués par LRI en fonction des paramètres de fabrication. Matériaux \& Techniques, 2010, 98 (2), p.151-163. 10.1051/mattech/2010019 . hal-01847797

\section{HAL Id: hal-01847797 \\ https://hal.science/hal-01847797}

Submitted on 6 Nov 2019

HAL is a multi-disciplinary open access archive for the deposit and dissemination of scientific research documents, whether they are published or not. The documents may come from teaching and research institutions in France or abroad, or from public or private research centers.
L'archive ouverte pluridisciplinaire $\mathbf{H A L}$, est destinée au dépôt et à la diffusion de documents scientifiques de niveau recherche, publiés ou non, émanant des établissements d'enseignement et de recherche français ou étrangers, des laboratoires publics ou privés. 


\title{
Optimisation de la résistance au cisaillement interlaminaire (RCIL) des matériaux composites fabriqués par LRI en fonction des paramètres de fabrication $\star$
}

\author{
A. Njionhou ${ }^{1,2}$, F. Berthet ${ }^{1,2}$ et B. Castanie ${ }^{1,3}$ \\ 1 Université de Toulouse; INSA, UPS, Mines Albi, ISAE; ICA (Institut Clément Ader), France \\ e-mail : anjionho@mines-albi.fr \\ 2 École des Mines Albi, Campus Jarlard, 81013 Albi Cedex 09, France \\ e-mail : florentin.berthet@mines-albi.fr \\ 3 INSA, 135 avenue de Rangueil, 31077 Toulouse, France \\ e-mail : bruno.castanie@isae.fr
}

Mots-clés :

Carbone NCF ; infusion de résine liquide ; paramètres de

fabrication ; plan d'expérience ; $\mathrm{RCIL}$; teneur en porosités

\section{Key words:}

Carbon Non-Crimp-Fabric; LRI; manufacturing parameters; experimental plan; ILSS; void content
Résumé - La technique de fabrication de composites par infusion de résine (LRI : liquid resin infusion) consiste en l'imprégnation d'une résine liquide à travers l'épaisseur d'une préforme sèche. La compréhension des phénomènes physiques se produisant au cours des procédés d'infusion de résine sous vide a permis d'identifier les paramètres de fabrication qui ont le plus d'effets sur la variation des propriétés mécaniques des structures composites mises en œuvre. Ces paramètres sont classés en trois groupes : la texture du renfort, la configuration du procédé et les températures du procédé. Un plan d'expérience a été conçu pour comprendre les relations qui existent entre les paramètres de fabrication et les propriétés mécaniques des composites infusés. Cette étude a pour finalité de proposer et d'optimiser les propriétés mécaniques des composites carbone quadri axiaux NCF (non-crimpfabric)/époxy RTM 6 en jouant sur les paramètres liés au procédé LRI. Dans cette recherche, les influences et les effets de ces paramètres sur la résistance au cisaillement interlaminaire (RCIL) et la fraction volumique en porosités $V_{p}$ des structures fabriquées sont analysés de manière statistique. L'étude a montré une croissance de $V_{p}$ en fonction de l'augmentation de la température du moule ainsi que de la température de réticulation de la résine. Le taux de porosités dans les composites infusés augmente lorsque le nombre de couches de carbone NCF constituant la préforme croît. Ce dernier résultat se vérifie en prenant en considération le nombre de couches de carbone NCF de manière unique comme paramètre d'influence, mais aussi lorsque celui-ci est en interaction avec le nombre de couches de tissu drainant la résine le long de la pièce au cours du processus d'infusion. Les résultats des analyses ont prouvé que les propriétés en cisaillement interlaminaire (RCIL) sont influencées par le taux de porosités du matériau.

\begin{abstract}
Optimization of Interlaminar Shear Strength (ILSS) of composites materials made by Liquid Resin Infusion (LRI) according to the manufacturing parameters. The liquid resin infusion (LRI) process is a composite manufacturing technique where liquid resin infuses through the thickness of the preform reinforcement. Understanding the physical phenomena occurring during vacuum infusion process (VIP) allowed to identify the manufacturing parameters that have the largest effects on the variation of mechanical properties of composite structures. These parameters are classified by tree sets: texture reinforcement, process configuration and process temperatures. An experimental plan was designed to identify the relationship between the manufacturing parameters and mechanical properties of infused composites. The goal of this study is to propose and optimize the mechanical properties of quadri axial carbon non-crimp-fabric (NCF)/epoxy RTM 6 composites according to the LRI process parameters. In this research, influences and effects of these parameters on the Interlaminar Shear Strength (ILSS) and
\end{abstract}

\footnotetext{
* Cet article a été soumis dans le cadre des journées Rotomoulage, Thermoformage et Soufflage 2009 (RTS 2009) qui se sont tenues à l'École des Mines d'Albi du 11 au 13 mars 2009.
} 
the void content $V_{\mathrm{p}}$ of structures made were analysed statistically. Results showed the increase of $V_{\mathrm{p}}$ depending on increasing of mould temperature and increasing of curing temperature of epoxy RTM 6 resin. Void content inside infused composites increases when, the number of carbon NCF layers which constitutes the preform reinforcement increases. This last result is confirmed with the consideration of the number of carbon NCF layers in a unique manner like a influenced process parameter. This result is verified also when the number of NCF layers is in interaction between number of high porous media (HPM) layers fabric. This fabric helps draining resin along the length of structure during the process. The analysis proved that the Interlaminar shear properties are influenced by void content.

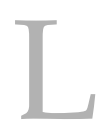

e procédé d'infusion de résine liquide, ou liquid resin infusion (LRI), est un procédé de fabrication de structures polymères renforcées de fibres pour des applications à hautes performances dans le secteur de l'aéronautique par exemple. Il est classé parmi les procédés d'infusion sous vide (VIP : vacuum infusion process) [1]. La figure 1 détaille les éléments du processus expérimental. En effet, la résine s'infuse à travers l'épaisseur d'une préforme sèche grâce à l'aspiration du vide. Le LRI est considéré comme un procédé à faible coût dans la fabrication de composites. Il ne nécessite pas de pression supplémentaire pour compacter la préforme ni pour injecter la résine. Le processus de réticulation de la résine lors de la fabrication est réalisé hors autoclave.

Une bâche à vide (ou membrane souple) est utilisée dans l'infusion comme moule supérieur. L'utilisation de cette bâche donne à ce procédé un coût d'outillage relativement faible lors de la fabrication de grandes structures par rapport à d'autres procédés qui utilisent des moules rigides. Un tissu drainant est utilisé (voir Fig. 1). C'est un milieu de distribution de résine ayant une perméabilité supérieure à celle de la préforme. Il facilite l'arrivée de la résine en surface le long de la pièce. Ensuite, la résine poursuit son écoulement dans l'épaisseur. La résine s'infuse donc grâce aux effets combinés du vide et des effets capillaires qui sont fonction du contact statique et dynamique de la résine avec les fibres du renfort. La vitesse d'écoulement de la résine dépend du gradient de pression atteint dans la cavité à vide (ensemble bâche à vide/moule). La perméabilité de l'empilement peut aussi jouer sur cette vitesse en fonction du nombre de couches de tissus considérées formant la préforme et du nombre de drainant utilisé. Les variabilités des propriétés finales des structures composites infusées de résine liquide sont donc causées certes en premier lieu par les propriétés intrinsèques des constituants (type de renfort et résine), mais aussi par le procédé d'infusion de résine en lui-même. Ces variabilités causées par le procédé sont entre autres dues aux configurations du montage d'infusion et aux températures utilisées lors du processus de fabrication. Il est par conséquent nécessaire d'identifier les paramètres du procédé LRI et de mesurer leurs effets en les quantifiant sur les propriétés mécaniques et la santé matière des composites mise en œuvre.

La première étape de cette recherche est d'identifier a priori grâce à une étude bibliographique les paramètres de fabrication qui ont le plus d'effets sur la variation des propriétés mécaniques des structures composites infusées. Il s'agira ensuite d'évaluer l'impact réel de ces paramètres - à savoir le nombre de couches de renfort, le nombre de couches de tissu drainant, les températures de moule, d'injection et de cuisson de résine - sur les propriétés des stratifiés carboneépoxy fabriqués par LRI. Dans ce travail, la position de la préforme dans le moule pendant le processus de fabrication (côté aspiration de vide ou côté injection) est aussi analysée.

La méthode d'étude des plans d'expérience est utilisée avant une analyse statistique des résultats. Dans cette publication, on se limitera à l'étude des variabilités de la résistance au cisaillement interlaminaire (RCIL) et de la fraction volumique en porosités $V_{\mathrm{p}}$ du stratifié infusé. L'utilisation de la microscopie électronique à balayage (MEB) permettra de faire le lien entre la microstructure et le comportement mécanique global. Enfin, une synthèse des résultats sera faite pour déterminer les meilleures conditions de fabrication afin d'optimiser les propriétés des composites fabriqués.

\section{Analyse expérimentale}

\section{Matériaux}

Deux stratifications en couches complémentaires de quatre plis unidirectionnels de fibres de carbone assemblées NCF (noncrimp-fabric), à module intermédiaire de 


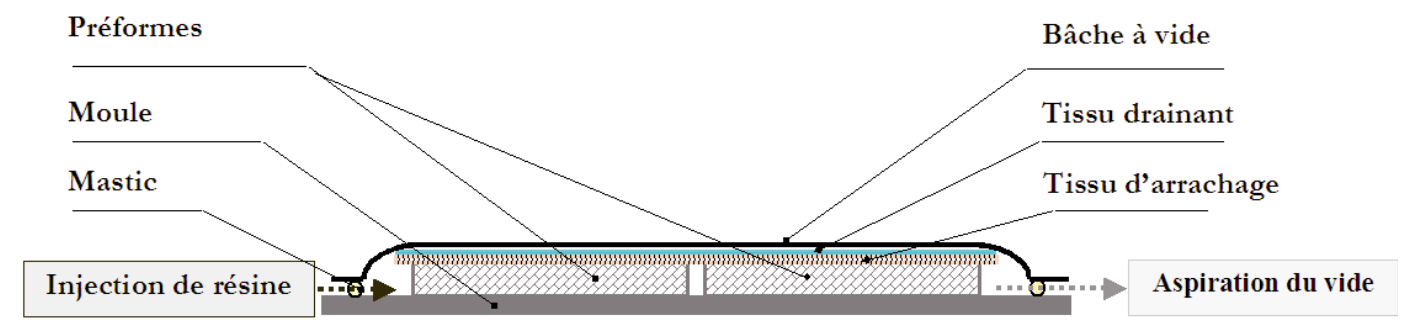

Fig. 1. Schéma expérimental du procédé L.R.I.

Fig. 1. Experimental setup of LRI process.

type Tenax IMS 60 E13 24K 830 tex, constituent le renfort. Ces tissus quadri axiaux (Fig. 2) ont une densité surfacique de $1088 \mathrm{~g} / \mathrm{m}^{2}$ et sont quasi-isotropes lors de leur drapage : $-45^{\circ} / 0^{\circ} /+45^{\circ} / 90^{\circ}$ et symétriquement $+45^{\circ} / 0^{\circ} /-45^{\circ} / 90^{\circ}$ (voir Fig. 3). Leur empilement forme les préformes lors de la fabrication des composites infusés. Ces tissus sont issus du commerce et fournis par SAERTEX. Ils sont conçus prioritairement pour de meilleures applications en résistance et ténacité ultimes [2].

La résine injectée est de l'époxy monocomposant de classe $180{ }^{\circ} \mathrm{C}$ type RTM 6 de HEXFLOW ${ }^{\circledR}$. C'est une résine pré-mixée de température de service comprise entre $-60^{\circ} \mathrm{C}$ et $180^{\circ} \mathrm{C}$. Des informations détaillées relatives à cette résine peuvent être trouvées dans la référence [3].

\section{Identification des paramètres de fabrication}

Les recherches déjà effectuées sur les autres procédés VIP ont permis d'identifier les principaux paramètres de fabrication qui se ramènent à l'infusion de résine liquide. Ce sont des paramètres qui ont le plus d'effets sur les performances et la qualité des structures composites infusées. Ils peuvent se classer en trois groupes.

Le premier groupe concerne la texture $d u$ renfort. On a pu identifier dans ce groupe la nature du poudrage se trouvant entre les plis des tissus, l'architecture de la préforme (tissage, tricotage, présence de renforts transverses : piquage ou couture) et en dernier lieu le drapage lui-même. Le taux ou le type de poudre contribue à la qualité du matériau et a des effets sur les propriétés du composite, en particulier à l'impact [4-7]. Le piquage de la préforme a un impact important sur la qualité et les propriétés mécaniques des composites, tant pendant le processus d'infusion par l'écoulement de la

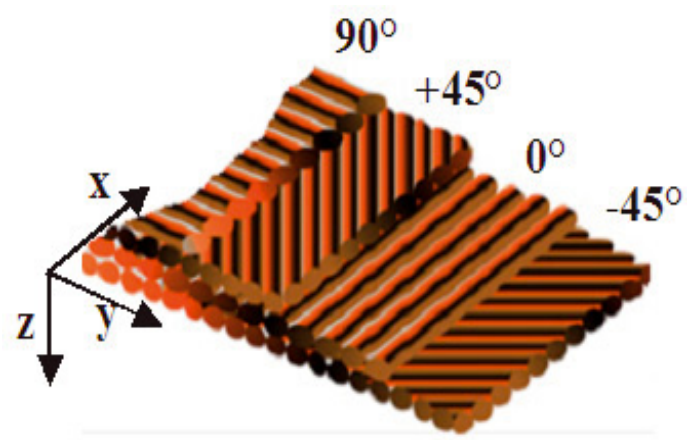

Fig. 2. Principe de fabrication des tissus de carbones quadri axiaux.

Fig. 2. Quadri-axial carbon fabrics manufacturing.

résine à travers les fibres transverses que lors de la polymérisation de celle-ci $[4,8-$ 11]. Pour cette étude, la poudre contenue dans la préforme est intrinsèque au renfort fourni par SAERTEX. Ce paramètre ne figure donc pas parmi les paramètres à analyser. Par ailleurs, GOVIGNON et al. ont montré l'évolution de l'épaisseur de la plaque lors $\mathrm{du}$ processus d'infusion. Ils ont également établi une relation entre la fraction volumique de fibres et la perméabilité en faisant intervenir le nombre de couches de renfort et l'épaisseur de la structure fabriquée [9]. Ce nombre de couches serait un paramètre $\mathrm{d}^{\prime}$ 'influence important car ses effets sur les propriétés mécaniques de la structure composite seraient soit directs, soit par interaction avec d'autres paramètres comme le nombre de couches de drainant. A priori, ses effets sont plus importants sur les propriétés en traction ou compression [5,12]. Dans cette étude, la texture du renfort, groupe 1 des paramètres de fabrication, est constituée par le nombre de couches de carbone NCF que forme la préforme. Les préformes peuvent contenir deux (2) ou quatre (4) couches de carbone NCF drapées selon le schéma donné à la figure 3. Les tissus de carbone NCF utilisés ont une perméabilité de $1 \times 10^{-12} \mathrm{~m}^{2}$. 


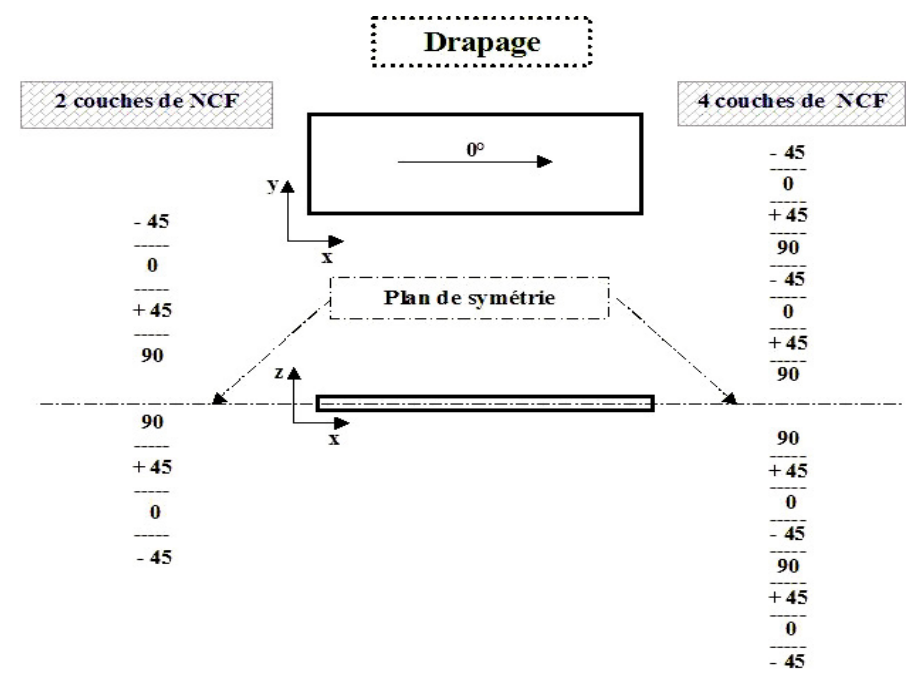

Fig. 3. Drapage des couches de carbone NCF.

Fig. 3. Laying-up of NCF carbon layers.

Le second groupe est la configuration du procédé. Plusieurs auteurs présentent le niveau de vide obtenu au cours de l'étape d'injection de résine dans le moulage des composites par voie liquide (LCM : liquid composite moulding) comme un paramètre primordial pour l'obtention d'une meilleure qualité des matériaux $[4,6,7,9,14]$. Ce niveau de vide représente la valeur de la pression absolue dans la cavité à vide (ensemble bâche à vide/moule : voir Fig. 1). Les manipulations effectuées lors des expériences sur le procédé LRI montrent que la réalisation d'un bon niveau de vide dépend de l'étanchéité obtenue dans la cavité au cours du processus de fabrication. Cette pression absolue n'est pas imposable et varie en fonction de l'aptitude au montage. Pour cette raison, le "niveau de vide", dont les valeurs atteintes lors de ces essais sont généralement inférieures à 2 mbar (1,5 mbar en moyenne), ne sera pas une variable pour l'analyse des paramètres dans la présente étude.

Par contre, la localisation de la structure par rapport aux points d'injection de résine est un facteur de variabilité des propriétés des composites. En effet, le champ des vitesses d'écoulement de la résine dans le moule dépend du lieu où se situe la préforme structurelle par rapport aux points et du nombre d'entrées et de sorties de résine. La variation de ce champ pourrait engendrée la formation de types, formes ou tailles de porosités dans le composite $[9,15,16]$. Elle influence par conséquent sur sa santé matière. Elle aurait a priori des effets sur sa fraction volumique en fibres et en porosités ainsi que sur ses caractéristiques mécaniques notamment en traction, compression et cisaillement interlaminaire [17-19]. Dans cette étude, le nombre de points d'entrée et de sortie ainsi que le type d'injection de résine sont fixes. Deux points périphériques sont utilisés. L'un est l'entrée de résine et l'autre le point d'aspiration de vide de la cavité. Pour une expérience donnée, deux structures composites seront fabriquées issues d'une préforme placée proche de l'injection de résine et une autre placée du côté de l'aspiration du vide vers la pompe (voir Fig. 1). Ces deux localisations seront prises en compte dans l'analyse. On utilisera comme paramètre du plan d'expérience la variable binaire côté aspiration/côté injection. Elle permettra d'évaluer la variation des propriétés mécaniques et la stabilité de l'épaisseur le long de la structure.

Par ailleurs, le tissu drainant est un paramètre caractéristique de l'écoulement de la résine pour la fabrication des structures composites par VIP [20]. Il est également identifié dans cette partie comme paramètre d'influence a priori. Sa présence dans la configuration ou son nombre pourrait avoir une influence sur les propriétés du composite soit de manière unique, soit par interaction avec le nombre de couches de fibres de renfort [12]. L'identification de ce paramètre comme facteur d'impact est récente. Son intégration dans l'analyse permettra de mieux maîtriser ses effets. Dans cette étude, le tissu drainant utilisé a une perméabilité de $4 \times 10^{-10} \mathrm{~m}^{2}$ et son nombre sera varié de un à deux.

Les températures $d u$ procédé constituent le troisième groupe. Il s'agit de la température du moule, de la température d'injection de résine et de la température de cuisson du stratifié. Ces températures ont une influence sur la viscosité de la résine et donc sur sa vitesse d'écoulement à travers la préforme $[4-7,9,11,14]$. Ces paramètres sont inclus dans l'étude en faisant varier leurs valeurs dans les plages admissibles des propriétés. La résine peut être injectée à $60^{\circ} \mathrm{C}$ ou $80^{\circ} \mathrm{C}$, le moule préchauffé à $100^{\circ} \mathrm{C}$ ou $120{ }^{\circ} \mathrm{C}$, et le stratifié peut être réticulé à $160^{\circ} \mathrm{C}$ ou $180^{\circ} \mathrm{C}$. Pour chacune des températures du procédé, la deuxième valeur prise est sa maximale admise par le constructeur de la résine [3].

\section{Définition du plan d'expérience}

La méthode des plans d'expérience vise à organiser de façon économique et rationnelle 
Tableau 1. Description du plan d'expérience.

Table 1. Description of design of experiments.

\begin{tabular}{|c|c|c|c|c|c|c|c|c|}
\hline \multicolumn{2}{|c|}{$\begin{array}{c}\text { Conditions de } \\
\text { fabrication }\end{array}$} & \multicolumn{7}{|c|}{$\begin{array}{c}\text { Paramètres du } \\
\text { procédé }\end{array}$} \\
\hline$\overline{\mathrm{N}^{\circ}}$ & Exp. & $\begin{array}{l}\text { Nombre de } \\
\text { couches de } \\
\text { N.C.F }\left(\mathrm{P}_{1}\right)\end{array}$ & $\begin{array}{l}\text { Nombre de } \\
\text { couches de } \\
\text { drainant }\left(\mathrm{P}_{2}\right)\end{array}$ & $\begin{array}{c}\text { Côté aspiration/ } \\
\text { Côté injection } \\
\left(\mathrm{P}_{3}\right)\end{array}$ & $\begin{array}{c}\text { Interaction } \\
\text { NCF et drainant } \\
\left(\mathrm{P}_{1} \mathrm{P}_{2}\right)\end{array}$ & $\begin{array}{c}\text { Temp. moule } \\
\left({ }^{\circ} \mathrm{C}\right) \\
\left(\mathrm{P}_{4}\right)\end{array}$ & $\begin{array}{c}\text { Temp. injection } \\
\left({ }^{\circ} \mathrm{C}\right) \\
\left(\mathrm{P}_{5}\right)\end{array}$ & $\begin{array}{c}\text { Temp. cuisson } \\
\left({ }^{\circ} \mathrm{C}\right) \\
\left(\mathrm{P}_{6}\right)\end{array}$ \\
\hline 1 & $5 \mathrm{~A}$ & 2 & 2 & Aspiration & 1 & 100 & 80 & 180 \\
\hline 2 & $5 \mathrm{I}$ & 2 & 2 & Injection & 1 & 100 & 80 & 180 \\
\hline 3 & $6 \mathrm{~A}$ & 2 & 1 & Aspiration & 2 & 120 & 80 & 160 \\
\hline 4 & $6 \mathrm{I}$ & 2 & 1 & Injection & 2 & 120 & 80 & 160 \\
\hline 5 & $7 \mathrm{~A}$ & 4 & 1 & Aspiration & 1 & 100 & 60 & 160 \\
\hline 6 & $7 \mathrm{I}$ & 4 & 1 & Injection & 1 & 100 & 60 & 160 \\
\hline 7 & $8 \mathrm{~A}$ & 4 & 2 & Aspiration & 2 & 120 & 60 & 180 \\
\hline 8 & $8 \mathrm{I}$ & 4 & 2 & Injection & 2 & 120 & 60 & 180 \\
\hline 9 & $9 \mathrm{~A}$ & 4 & 2 & Aspiration & 2 & 110 & 70 & 170 \\
\hline 10 & $9 \mathrm{I}$ & 4 & 2 & Injection & 2 & 110 & 70 & 170 \\
\hline
\end{tabular}

une expérience dépendant de plusieurs paramètres. Cette méthode permet d'assurer la qualité des résultats de l'expérience, tout en limitant au maximum le nombre des essais à réaliser La méthode utilisée est celle de la table de Taguchi L8 [4,21,22]. Elle est constituée d'un tableau composé de sept colonnes de facteurs qui sont les paramètres du procédé identifié précédemment $\left(P_{k}\right)$, de huit lignes principales et deux lignes de vérification de l'analyse des résultats (lignes Nos. 9 et 10) qui représentent les conditions de fabrication. Dans le tableau, chaque facteur possède deux niveaux de variation. À partir de ce plan, la mise en œuvre des composites de renforts en fibres de carbone NCF et de matrice époxy RTM 6 a été fait selon dix conditions expérimentales différentes.

\section{Fabrication des plaques}

Le dispositif de fabrication des structures composites carbone NCF/époxy RTM 6 infusées est constitué d'un moule carré en aluminium de $500 \times 500 \mathrm{~mm}$, d'une table chauffante programmable ayant une température maximale de $250{ }^{\circ} \mathrm{C}$, d'un pilote d'injection de résine (RTM ISOJET) équipée d'une pompe à vide, d'un pot de résine sur balance et d'un ordinateur réalisant le pilotage d'infusion (chauffage, dégazage, injection de résine, mise sous vide du moule) et l'acquisition des données (températures et pressions à temps réel, masse de résine injectée).

Les tissus en fibres de carbone NCF sont découpés et drapés en deux parties de $400 \times 150 \mathrm{~mm}$ avec le nombre de couches indiqué par la condition de fabrication choisie (Tab. 1). Ils sont disposés en deux préformes à température ambiante sur le moule posé sur la table chauffante. L'une est proche du point d'aspiration du vide et l'autre de celui d'injection de résine. Un agent démoulant semi-permanent (FREKOTE) est appliqué au préalable sur la surface du moule afin de faciliter le démoulage du composite après refroidissement. Les préformes sont espacées l'une de l'autre d'une distance de $100 \mathrm{~mm}$ maximum environ pour permettre la disposition en bord de moule des éléments d'étanchéité et de mise sous vide de l'enceinte (Fig. 4). Un tissu d'arrachage pour faciliter le démoulage, un ou deux tissus drainants et une bâche à vide recouvrent l'ensemble et créent ainsi son étanchéité par collage de la bâche tout autour du moule sur un joint de mastic.

Avant de procéder à la mise sous vide de l'ensemble, on programme le chauffage du moule et de la résine de façon à ce que cela se fasse de manière simultanée. Ces programmations sont faites successivement à la table chauffante et au pilote d'injection. Des thermocouples sont placés, non pas à l'intérieur du composite par souci de préserver sa qualité et son étanchéité, mais à la surface de la bâche sur les préformes afin de vérifier les températures réelles au cours du processus (Fig. 4). Lorsque les températures escomptées sont atteintes (température de moule : 100 ou $120{ }^{\circ} \mathrm{C}$ mesurée sur la table et température de résine : 60 ou $80^{\circ} \mathrm{C}$ mesurée à l'aide d'une sonde placée dans le pot de résine), il se passe successivement le dégazage de la résine sous vide d'air pendant 10 min et la mise sous vide de l'enceinte bâche à vide/moule pendant $10 \mathrm{~min}$ (niveau de vide en moyenne inférieur à 2 mbar prélevé à la pompe pour toutes les fabrications). Lorsque le conduit d'alimentation atteint la température d'injection de résine prévue, le 

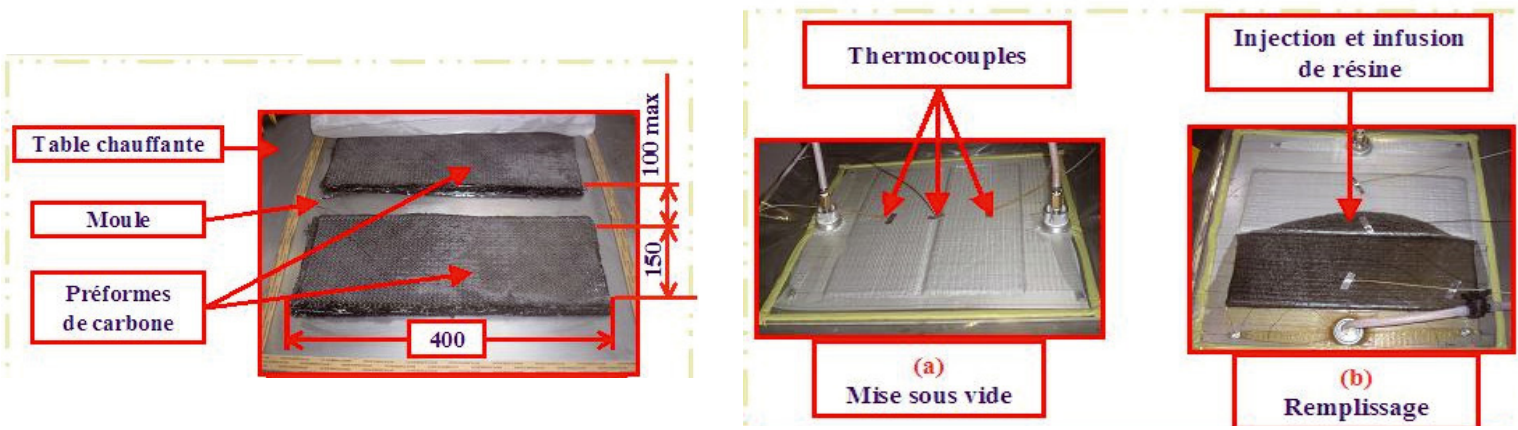

Fig. 4. Fabrication de composites NCF/RTM6.

Fig. 4. Manufacturing of NCF carbon and RTM 6 epoxy resin infused composites.

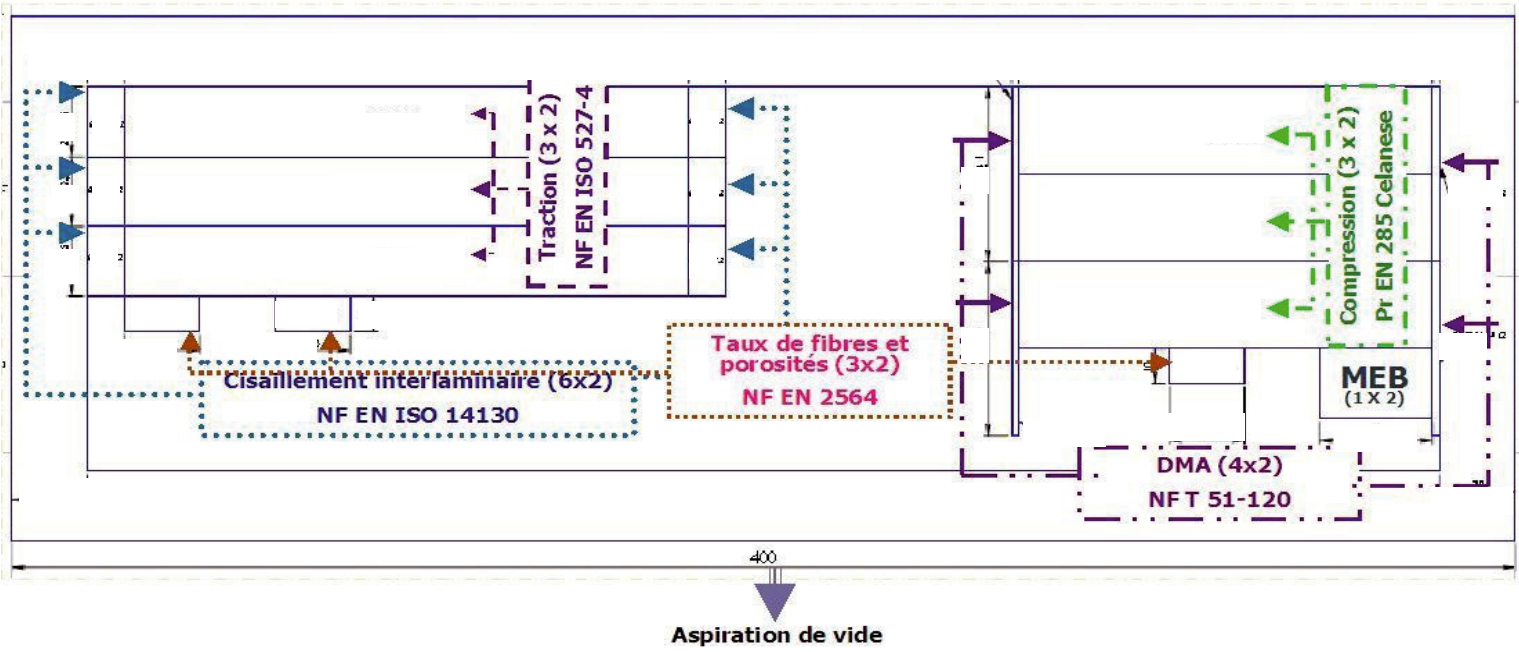

Fig. 5. Plan de découpe des composites infusés 2 NCF/epoxy RTM 6.

Fig. 5. Sampling plan of infused composites of 2 NCF layers and RTM 6 epoxy resin.

Tableau 2. Cycle de cuisson de structures composites carbone NCF/RTM 6.

Table 2. Curing cycle of NCF carbon and RTM 6 epoxy composite structures.

\begin{tabular}{cccc}
\hline $\begin{array}{c}\text { Temérature de } \\
\text { cuisson }\left({ }^{\circ} \mathrm{C}\right)\end{array}$ & $\begin{array}{c}\text { Temps de } \\
\text { montée }(\text { min.) }\end{array}$ & $\begin{array}{c}\text { Temps de } \\
\text { maintien }(\text { min.) }\end{array}$ & $\begin{array}{c}\text { Temps de } \\
\text { descente }(\text { min.) }\end{array}$ \\
\hline 160 & 45 & 75 & 120 \\
170 & 60 & 70 & 120 \\
180 & 60 & 60 & 120 \\
\hline
\end{tabular}

processus se poursuit ensuite par son injection et son infusion dans la préforme. La résine pénètre d'abord dans le(s) tissu(s) drainant(s) puis s'infuse dans l'épaisseur de la préforme structurelle.

L'infusion se poursuit jusqu'à la sortie de la résine au point d'aspiration du vide. Le même niveau de vide est maintenu de la phase d'injection au remplissage complet de l'enceinte. Il est nécessaire de s'assurer que la résine ne comporte pas de bulles. L'enceinte est ensuite isolée du pilote (entrée et sortie de résine fermée) pour procéder à la polymérisation. Elle se fait à température de cuisson prévue (160 ou $180{ }^{\circ} \mathrm{C}$ ) sur la table chauffante programmable suivant un cycle de cuisson (Tab. 2) avant refroidissement. Après la polymérisation de la résine, chaque plaque composite est refroidie pendant une durée minimale de $2 \mathrm{~h}$ afin d'obtenir une pièce suffisamment rigide avant son démoulage et son nettoyage. Une fois la plaque composite obtenue, il faut la découper aux dimensions des éprouvettes (Fig. 5) en fonction des essais de caractérisation à faire.

\section{Essais}

Six éprouvettes sont prélevées sur chaque plaque composite fabriquée et testées en $c i$ saillement interlaminaire. L'essai se présente sous la forme d'un montage en flexion trois points avec appuis rapprochés (Fig. 6). Il est décrit par la norme NF EN ISO 14130 [13]. Les éprouvettes ont des dimensions moyennes de $20 \times 10 \times 2 \mathrm{~mm}$ pour les plaques à deux couches de NCF et de $40 \times 20 \times 4 \mathrm{~mm}$ pour celles à quatre couches. 


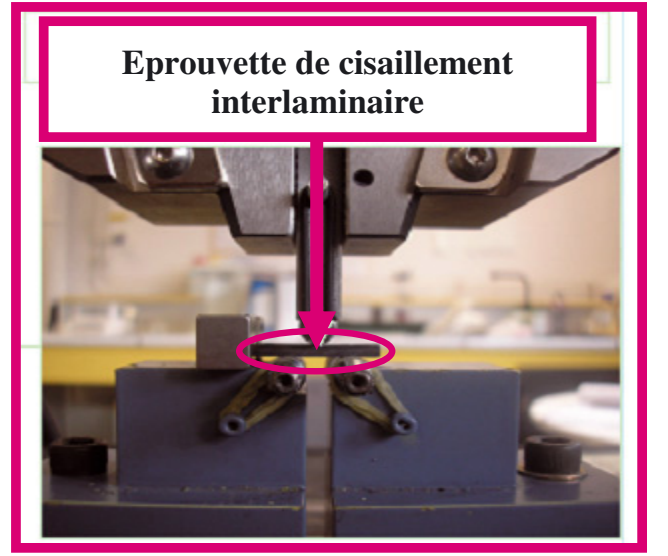

Fig. 6. Montage pour essai de cisaillement interlaminaire.

Fig. 6. Interlaminar shear test.

La caractérisation du taux de porosités des matériaux est faite sur trois éprouvettes prélevées sur chaque plaque composite (voir plan de découpe des éprouvettes à la Fig. 5). Ces éprouvettes sont de dimensions moyennes respectives $20 \times 10 \times 2 \mathrm{~mm}$ et $20 \times 5 \times 4 \mathrm{~mm}$ pour les deux et quatre couches de NCF selon la norme NF EN 2564. Il s'agit du couplage des méthodes de détermination de la masse volumique par immersion dite à la balance hydrostatique (NF T 51-063 ou norme internationale ISO 1183) et de la teneur en fibres et en résine par dissolution et par décantation (NF T 57-608 ou norme internationale ISO CD 11667-3) des plastiques renforcés de fibres usinés ou finis [23]. La fraction volumique en porosités $V_{\mathrm{p}}$ du matériau est donc la différence entre $100 \%$ de volume initial de matière et la somme des fractions volumiques en fibres $V_{\mathrm{f}}$ et en résine $V_{\mathrm{r}}$ (Éq. (1)).

$$
V_{\mathrm{p}}=100-\left(V_{\mathrm{f}}-V_{\mathrm{r}}\right)
$$

\section{Résultats et discussion}

Le tableau 3 énumère les résultats moyens des caractérisations des composites par condition de fabrication. Chaque caractérisation a été faite de la même manière sur toutes les plaques et suivant chaque configuration. Toutes les éprouvettes ont été prélevées exactement au même endroit suivant leur plan de découpe (Fig. 5).

\section{Analyse statistique}

$$
\begin{aligned}
y= & c+\left[a_{1}, a_{2}\right] P_{1}+\left[b_{1}, b_{2}\right] P_{2}+\left[c_{1}, c_{2}\right] P_{3} \\
& +\left[\begin{array}{ll}
(a b)_{11} & (a b)_{12} \\
(a b)_{21} & (a b)_{22}
\end{array}\right] P_{1} P_{2}+\left[d_{1}, d_{2}\right] P_{4} \\
& +\left[e_{1}, e_{2}\right] P_{5}+\left[f_{1}, f_{2}\right] P_{6}
\end{aligned}
$$

L'analyse des résultats se préoccupe de déterminer parmi les paramètres énumérés dans le plan d'expérience ceux qui ont des effets sur les variations des quantités caractérisées. Elle consiste à faire une modélisation des données et des calculs des effets des facteurs (paramètres) sur la réponse (résultats). Le principe est le même que celui décrit à la référence [22] en ce qui concerne la modélisation à "modèle avec interactions". Les calculs sont faits à l'aide de l'outil d'analyse «régression linéaire 》 de la commande « utilitaire d'analyse » dans Excel. Le modèle général est une expression linéaire de la variable réponse $Y$ (qui peut être ici $R C I L$ ou $V_{\mathrm{p}}$ ) en fonction des paramètres $P_{k}$ ( $k$ varie de 1 à 6) et des interactions qu'on a supposés comme ayant des effets sur la réponse (voir Sects. 2.2 et 2.3). Elle peut s'écrire dans notre cas selon l'expression de l'équation (2), avec $C$ la constante du modèle, et $a_{i}, b_{i}, c_{i}, d_{i}$, $e_{i}$, et $f_{i}$ les coefficients du modèle associés aux paramètres $P_{k}$ du tableau $1 . i$ représente les niveaux de variation des facteurs - il varie de 1 à 2 - et $P_{1} P_{2}$ représente l'interaction entre le paramètre $P_{1}$ et $P_{2}$.

Les coefficients trouvés après calcul à l'aide du programme de traitement de données quantifient ainsi les effets des paramètres. L'analyse des résultats consiste donc à rechercher le meilleur modèle du plan. C'est le modèle contenant uniquement les paramètres dont les effets sont statistiquement significatifs. Un paramètre est considéré comme ayant des effets significatifs $(S)$ sur le résultat lorsque la probabilité de nullité $\alpha$ du coefficient du paramètre, dans le tableau de l'analyse de la variance, est inférieure ou égale à $5 \%(\alpha \leq 5 \%)$. Si $\alpha<1 \%$, le paramètre est très significatif (TS). Il est peu significatif (PS) quand la probabilité est comprise entre 5 et $10 \%(5 \%<\alpha<10 \%)$ et non significatif (NS) dans les autres cas.

Les paragraphes qui suivent présenteront les paramètres jugés statistiquement significatifs ainsi que les valeurs de leurs effets sur les réponses de caractérisation.

\section{Effets sur la résistance au cisaillement interlaminaire}

Des essais de cisaillement interlaminaire ont été faits sur les composites infusés fabriqués et les résultats obtenus ont été analysés. L'analyse statistique a permis d'identifier le meilleur modèle associé à la résistance au cisaillement interlaminaire (voir Sect. « Analyse statistique» pour la définition). Il 
Tableau 3. Synthèse des résultats.

Table 3. Summary results.

\begin{tabular}{cccccccc}
\hline $\mathrm{N}^{\circ}$ & Exp. & $\begin{array}{c}\text { Taux de fibres } \\
(\%)\end{array}$ & $\begin{array}{c}\text { Ecart type } \\
\text { (3 éprouvettes) }\end{array}$ & $\begin{array}{c}\text { Taux de } \\
\text { porosités }(\%)\end{array}$ & $\begin{array}{c}\text { Ecart type } \\
\text { (3 éprouvettes) }\end{array}$ & $\begin{array}{c}\text { RCIL } \\
(\mathrm{MPa})\end{array}$ & $\begin{array}{c}\text { Ecart type } \\
\text { (6 éprouvettes) }\end{array}$ \\
\hline 1 & $5 \mathrm{~A}$ & 59,95 & 0,20 & 1,22 & 0,35 & 35,53 & 2,28 \\
2 & $5 \mathrm{I}$ & 58,96 & 0,93 & 0,51 & 0,08 & 36,12 & 1,78 \\
3 & $6 \mathrm{~A}$ & 56,77 & 0,84 & 0,36 & 0,06 & 40,24 & 1,42 \\
4 & $6 \mathrm{I}$ & 55,31 & 0,63 & 0,41 & 0,09 & 40,40 & 3,53 \\
5 & $7 \mathrm{~A}$ & 59,81 & 0,80 & 0,21 & 0,18 & 42,82 & 4,16 \\
6 & 7 I & 59,71 & 0,76 & 0,56 & 0,08 & 41,65 & 5,34 \\
7 & $8 \mathrm{~A}$ & 56,20 & 0,66 & 2,47 & 0,79 & 30,53 & 4,74 \\
8 & $8 \mathrm{I}$ & 53,83 & 0,59 & 3,95 & 1,34 & 32,55 & 3,77 \\
9 & $9 \mathrm{~A}$ & 61,47 & 0,43 & 1,44 & 0,27 & 35,37 & 3,44 \\
10 & $9 \mathrm{I}$ & 61,84 & 3,80 & 2,03 & 0,03 & 39,51 & 4,03 \\
\hline
\end{tabular}

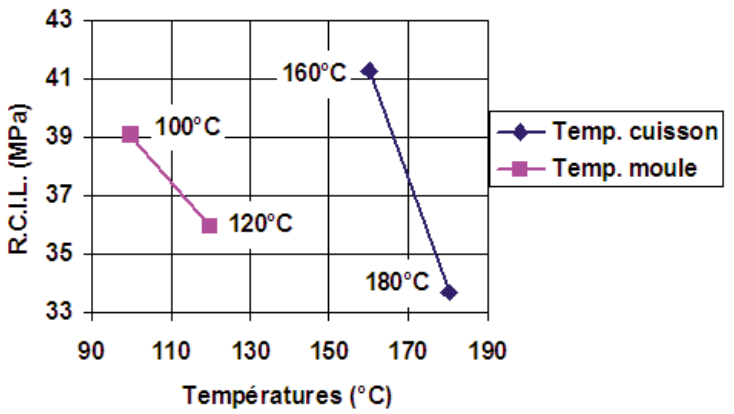

Fig. 7. Effets de la température de cuisson et de la température du moule sur la résistance au cisaillement interlaminaire de composites carbone NCF/époxy RTM 6.

Fig. 7. Effects of curing temperature and mould temperature on Interlaminar shear strength of NCF carbon and RTM 6 epoxy composites.

comporte les paramètres ci-dessous cités par ordre d'influence décroissante :

- la température de cuisson;

- la température du moule ;

- l'interaction entre le nombre de couches de NCF et le nombre de couches de drainant.

Ces paramètres sont ceux du plan d'expérience (Sect. "Définition du plan d'expérience » : Tab. 1) qui ont le plus d'influence sur la variation de la RCIL. L'analyse de la variance pour ce modèle donne des probabilités $\alpha_{T \text { cuisson égale à } 1,0925 \times 10^{-9}, \alpha_{T \text { moule }}}$ égale à $4,1567 \times 10^{-9}$ et $\alpha_{\text {Int.NCF/drainant égale }}$ à $4,0572 \times 10^{-8}$. Les effets des trois facteurs cités sont marqués très significatifs (TS) sur les variabilités de la réponse $(\alpha<1 \%)$.

La figure 7 présente les variations de la résistance au cisaillement interlaminaire des composites carbone NCF/époxy RTM 6 en fonction des valeurs des températures de cuisson et de moule choisies. La RCIL des composites diminue de $8 \mathrm{MPa}$ environ

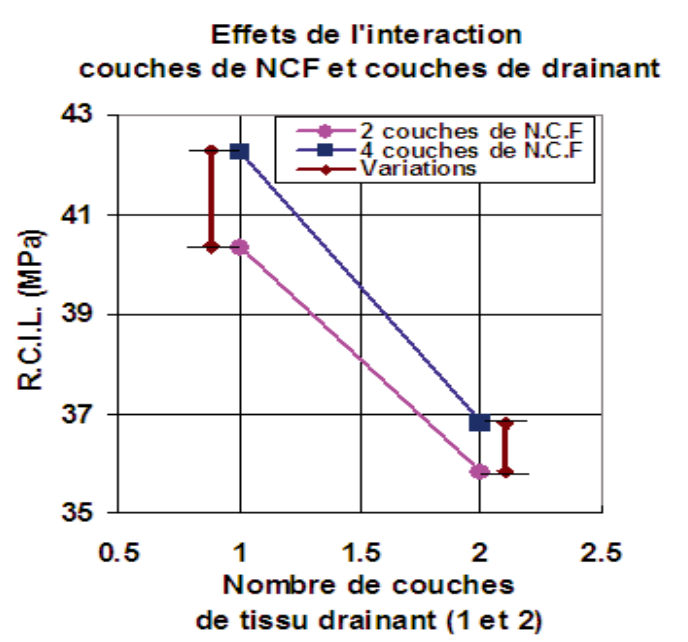

Fig. 8. Effets de l'interaction nombre de couches de N.C.F et de tissu drainant sur la résistance au cisaillement interlaminaire de composites carbone NCF/époxy RTM 6.

Fig. 8. Effects of the interaction between number of NCF layers and High Porous Medias (HPM) layers on Interlaminar shear strength of NCF carbon and RTM 6 epoxy composites.

lorsque la température de cuisson augmente de 160 à $180^{\circ} \mathrm{C}$. Cette baisse de la RCIL est aussi constatée pour les stratifiés fabriqués avec des températures de moule de $120^{\circ} \mathrm{C}$ par rapport à ceux produits avec des températures de moule de $100{ }^{\circ} \mathrm{C}$ et est de $3 \mathrm{MPa}$ en moyenne environ pour ces facteurs. L'effet du taux de fibres sur la RCIL n'a pas été significatif sur les tests effectués.

L'analyse révèle aussi une sensibilité de la résistance au cisaillement due à la présence de deux ou quatre couches de carbone NCF avec une ou deux couches de tissu drainant (Fig. 8). On constate dans l'ensemble que l'utilisation de deux couches de drainant au lieu d'une seule fait chuter la RCIL quelque soit le nombre de couches de carbone NCF utilisé. Il paraît judicieux 
d'analyser la microstructure des matériaux fabriqués afin de mieux comprendre cette variation. Cette analyse sera détaillée après celle des effets sur le taux de porosités au paragraphe «Analyse de la microstructure».

\section{Effets sur le taux de porosités}

Quatre paramètres ont été trouvés par l'analyse statistique comme affectant la variation du taux de porosités dans les composites époxy RTM 6 renforcés de carbone NCF infusés. L'ensemble de ces paramètres constitue le meilleur modèle du plan associé à cette réponse (voir Sect. «Analyse statistique»). Ils se classent par ordre d'influence décroissante de la manière suivante :

- la température de cuisson;

- le nombre de couches de carbone NCF ;

- la température du moule ;

- l'interaction entre le nombre de couches de NCF et le nombre de couches de drainant.

La figure 9 illustre une partie des résultats de l'analyse. L'augmentation de la température de cuisson, de la température du moule ou du nombre de couches de carbone NCF provoque la croissance de la teneur en vide dans les composites infusés. En effet, les composites ayant deux couches de NCF possèdent des fractions volumiques en porosités $V_{\mathrm{p}}$ inférieures de $0,91 \%$ en valeur absolue par rapport à ceux à quatre couches. Une variation est observée aussi dans les composites fabriqués avec des températures de moule de 100 au lieu de $120^{\circ} \mathrm{C}$. Pour l'augmentation de ce facteur, $V_{\mathrm{p}}$ croît en moyenne de 0,45 à $1,80 \%$. La hausse de la température de cuisson de 160 à $180{ }^{\circ} \mathrm{C}$ engendre une croissance moyenne de $V_{\mathrm{p}}$ d'environ 1,65\%.

L'augmentation du taux de porosités dans un composite infusé affecte sa qualité. Pour ce paramètre, on estime que pour les composites fabriqués selon les procédés VIP, il faut que $V_{\mathrm{p}}$ soit inférieur à $1,07 \%$ [24]. Ce critère amène vers le quatrième paramètre d'influence identifié dans l'analyse qui est l'interaction entre le nombre de couches de carbone NCF et le nombre de couches de tissu drainant. En effet, on peut observer à la figure 10 que $V_{\mathrm{p}}$ varie en fonction de la combinaison entre le nombre de couches de NCF utilisé et le nombre de tissus drainants. À la configuration 1, la condition de fabrication est 1 couche de drainant et 4 couches de NCF. À la configuration 3 , on a le même nombre de drainant pour 2 couches de NCF. Pour

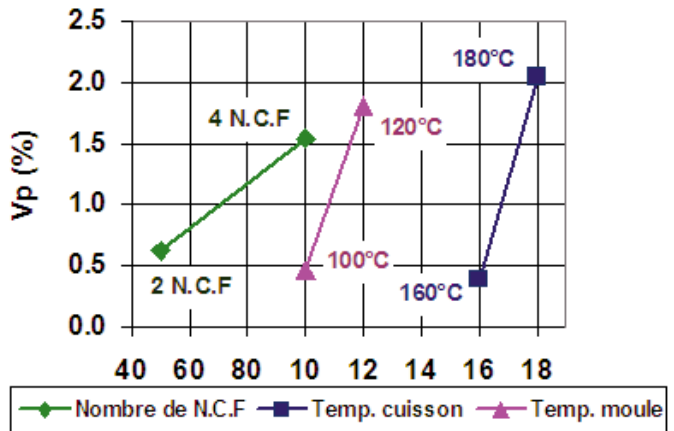

Fig. 9. Effets du nombre de couches de NCF, de la température de cuisson et de la température de moule sur la fraction volumique en porosités de composites carbone NCF/époxy RTM 6.

Fig. 9. Effects of the number of NCF layers, curing temperature and mould temperature on the void content of NCF carbon and RTM 6 epoxy composites.

Effets de l'Interaction:

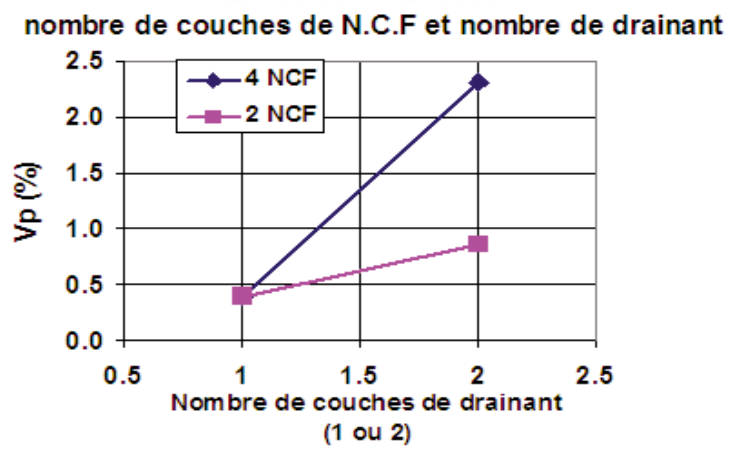

Fig. 10. Effets de l'interaction entre le nombre de couches de NCF et le nombre de drainant sur la fraction volumique en porosités de composites carbone NCF/époxy RTM 6.

Fig. 10. Effects of the interaction between number of NCF layers and High Porous Medias (HPM) layers on the void content of NCF carbon and RTM 6 epoxy composites.

ces deux conditions de fabrication, en plus de la condition 4 qui est 2 tissus drainants et 2 couches de $\mathrm{NCF}$, on trouve $V_{\mathrm{p}}$ inférieur à $1 \%$. D'après le critère de qualité énoncé, les composites issus de ces configurations sont corrects. Ceux issus de la configuration 2 (2 tissus drainants et 4 couches de NCF) ont une moindre qualité.

Au bilan, on a pu constater des variations de taux de porosités des composites infusés d'une configuration à une autre qui pourraient a priori s'expliquer par l'analyse de leur microstructure.

\section{Analyse de la microstructure}

Cette partie s'intéresse à l'observation au microscope électronique à balayage (MEB) et à l'analyse de la microstructure des composites dans chacune des quatre configurations 


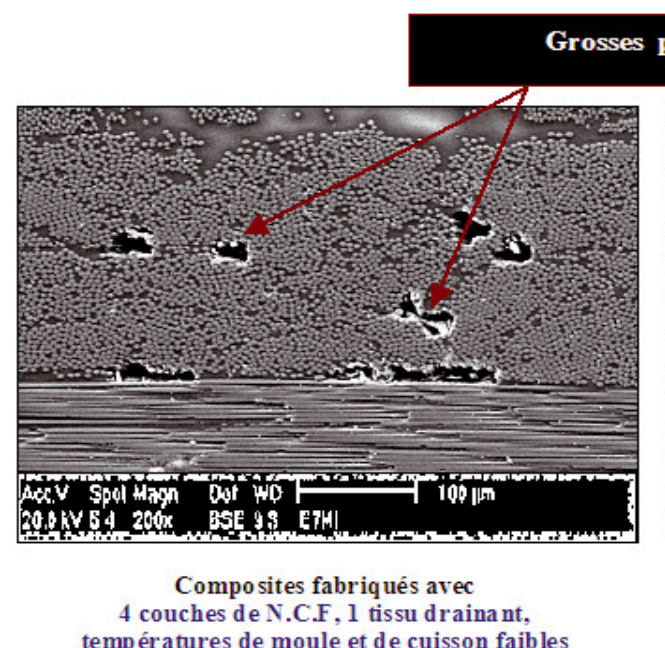
porosités

Fig. 11. Analyse de la microstructure des composites infusés à 4 couches de carbone NCF. Fig. 11. Microstructure analysis of 4 NCF carbon layers infused composites.

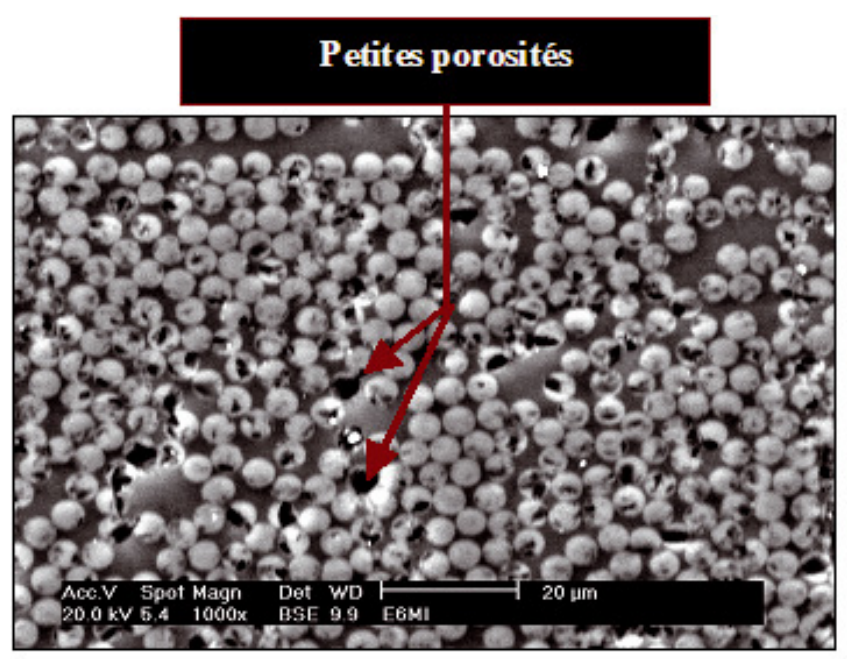

Composites fabriqués avec

2 couches de N.C.F, 1 tissu drainant, Températures de cuisson faibles

Fig. 12. Analyse de la microstructure des composites infusés à 2 couches de carbone NCF (configuration 3 ).

Fig. 12. Microstructure analysis of 2 NCF carbon layers infused composites (configuration 3).

énoncées dans la Section «Effets sur le taux de porosités ».

D'une manière générale, les composites fabriqués à l'aide de 4 couches de carbone NCF présentent des porosités dites de « grosses tailles » de l'ordre de 30 à $100 \mu \mathrm{m}$ (Fig. 11). Mais les observations faites sur les stratifiés issus des configurations 1 et 2 (Tab. 1) montrent que les porosités sont de tailles croissantes sur les configurations à 2 tissus drainants et avec des températures de moule et de cuisson élevées. Elles sont moins importantes dans les configurations avec 1 tissu drainant et obtenues avec des

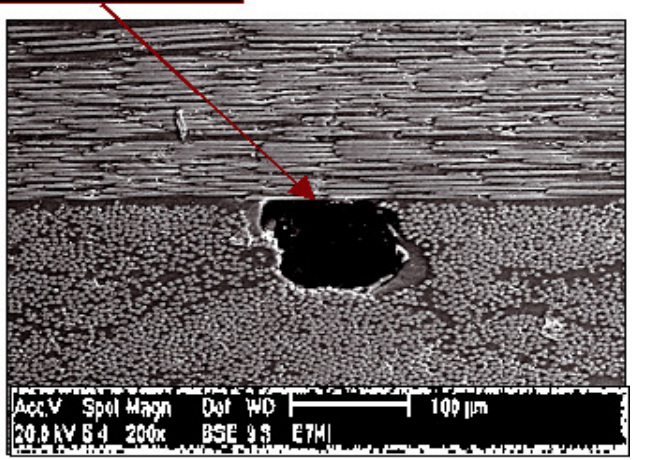

Composites fabriqués avec

4 couches de N.C.F, 2 tissus drainant, températures de moule et de cuisson élevées températures de moule et de cuisson faibles. Ceci expliquerait et confirmerait les résultats trouvés en RCIL : valeurs basses lors de l'utilisation des températures élevées (Fig. 7) mais aussi avec 2 tissus drainants (Fig. 8).

Les observations au MEB des composites à 2 couches de NCF présentent des porosités dont les diamètres sont inférieurs à $10 \mu \mathrm{m}$. Elles sont de «petites tailles » dans l'ensemble et assez régulières lorsque ces composites sont faits avec 1 tissu drainant et des températures de cuisson faibles (Fig. 12).

Pour ces mêmes composites à 2 couches de NCF, lorsqu'ils sont fabriqués avec 2 tissus drainants et des températures de cuisson élevées, on observe des grosses porosités (Fig. 13) entre les plis des couches de carbone NCF (entre le pli à $0^{\circ}$ et celui à $-45^{\circ}$ par exemple) ainsi que dans certains plis (celui à $-45^{\circ}$ par exemple).

Ces dernières observations expliquent la légère augmentation du taux de porosités selon la configuration 4 (Fig. 10). Elles clarifieraient aussi le résultat selon lequel la valeur moyenne la plus faible de la résistance au cisaillement interlaminaire serait dans cette configuration (Fig. 8).

Nous pouvons conclure ces analyses microscopiques en affirmant qu'une augmentation de température peut entraîner une croissance du taux de porosités et des contraintes internes dans les composites [17, 18,25-27] et ainsi détériorer sa qualité mécanique.

\section{Synthèse des résultats}

Les analyses statistiques des relations entre les paramètres de fabrication des composites 


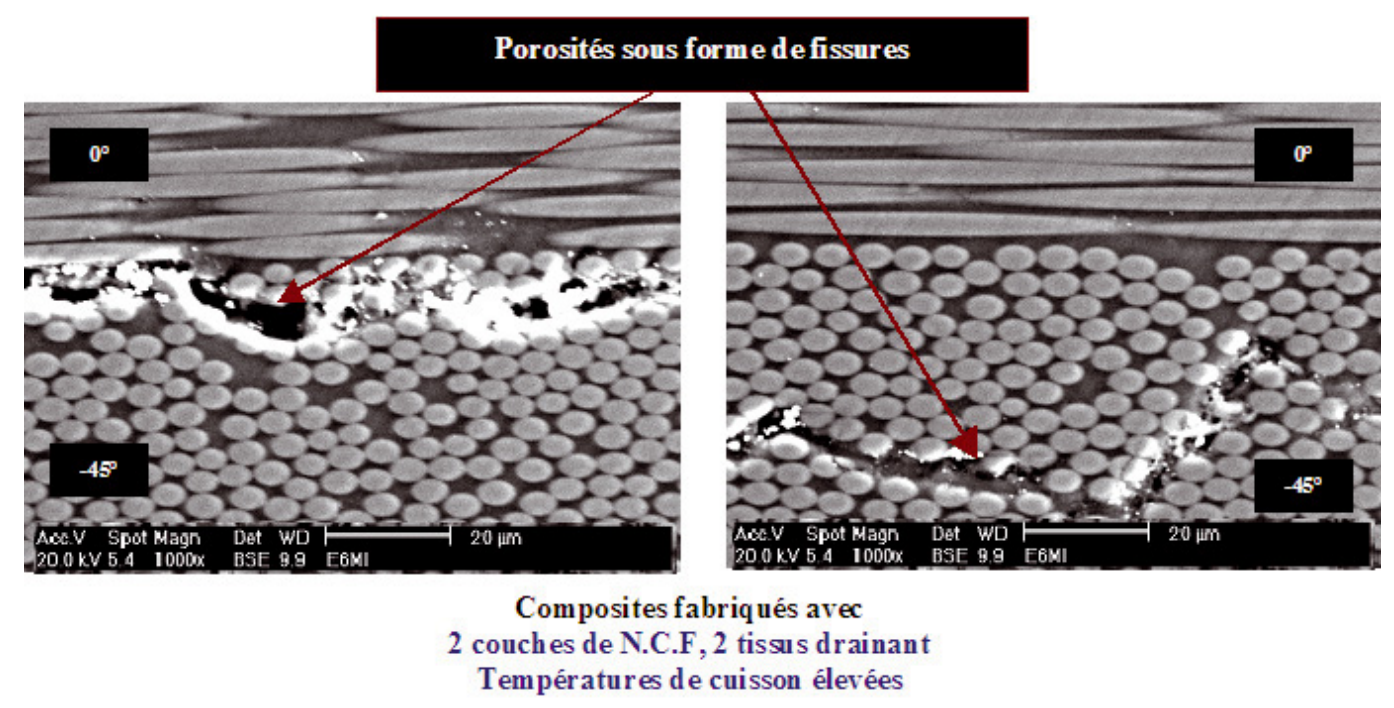

Fig. 13. Analyse de la microstructure des composites infusés à 2 couches de carbone NCF (configuration 4).

Fig. 13. Microstructure analysis of 2 NCF carbon layers infused composites (configuration 4).

mis en œuvre par le procédé LRI et leur propriété en cisaillement interlaminaire ont prouvé qu'il existe des effets de la température de moule, de la température de cuisson et de l'interaction entre le nombre de couches de carbone NCF et le nombre de couches de tissu drainant sur la variation de la résistance au cisaillement interlaminaire (RCIL). Ces mêmes paramètres, en plus du nombre de couches de carbone NCF analysé comme paramètre influent de manière unique, ont aussi un impact sur la fraction volumique en porosités $V_{\mathrm{p}}$ de ces composites.

La valeur du taux de porosités d'un matériau composite est un facteur de variation de sa qualité et de sa santé matière. Les observations microscopiques et l'analyse de la microstructure des matériaux fabriqués ont justifié les résultats trouvés précédemment lors des analyses des réponses de caractérisation. Elles ont permis d'établir des explications à ces résultats. Le tableau 4 et la figure 14 illustrent la relation qui existe entre la RCIL et $V_{\mathrm{p}}$. Cette relation est issue des analyses faites en fonction de la qualité atteinte du matériau. En effet, les RCIL des matériaux fabriqués sont meilleures lorsque $V_{\mathrm{p}}$ est inférieur à $1 \%$.

En regardant le plan d'expérience de manière globale (Figs. 14 et 15), on trouve que les composites qui ont les meilleures propriétés en cisaillement interlaminaire sont ceux dont les taux de porosités sont les plus faibles. Ainsi, on peut déceler les meilleures conditions de fabrication des structures composites à base de fibres de carbone NCF et de résine époxy RTM 6 fabriquées par LRI

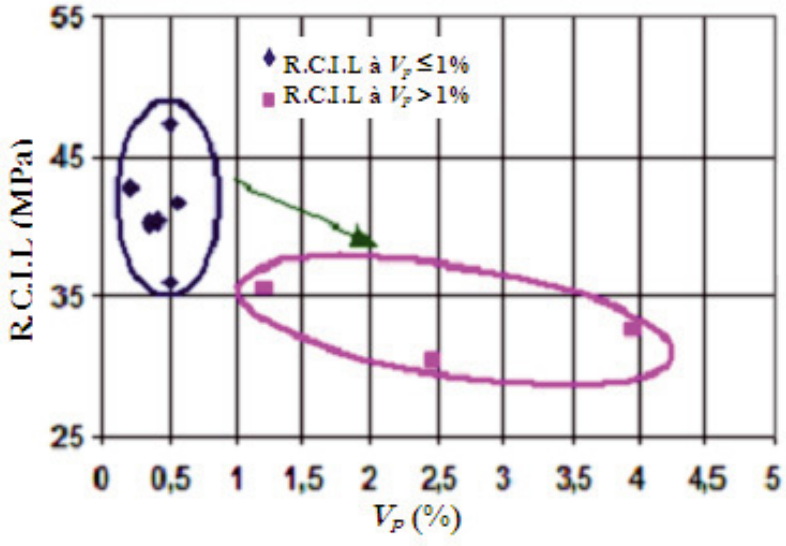

Fig. 14. Variation de la résistance au cisaillement interlaminaire en fonction de la fraction volumique en porosités.

Fig. 14. Variation of interlaminar shear strength according to the void content.

et sollicitées en cisaillement interlaminaire. L'utilisation de deux tissus drainants et des températures de moule et de cuisson élevées n'est pas conseillée pour ce type de composites (voir Sect. "Identification des paramètres de fabrication »: Tab. 1 pour les conditions de fabrication).

En revanche, les composites fabriqués avec un seul tissu drainant et des faibles températures de moule et de cuisson sont ceux donnant des meilleures propriétés en cisaillement interlaminaire.

\section{Conclusion}

Les paramètres du procédé de fabrication de composites par infusion de résine liquide 
Tableau 4. Relation entre la résistance au cisaillement interlaminaire et la fraction volumique en porosités des composites carbone NCF et époxy RTM 6.

Table 4. Relationship between Interlaminar shear strength and void content of NCF carbon and RTM 6 epoxy composites.

\begin{tabular}{lcc}
\hline & Fraction volumique en porosités & Résistance au cisaillement interlaminaire \\
& $V_{p}$ & R.C.I.L \\
\hline Qualité de & $V_{p} \leq 1 \%$ & Meilleures R.C.I.L \\
\cline { 2 - 3 } matériaux & $V_{p}>1 \%$ & Faibles R.C.I.L \\
\hline
\end{tabular}

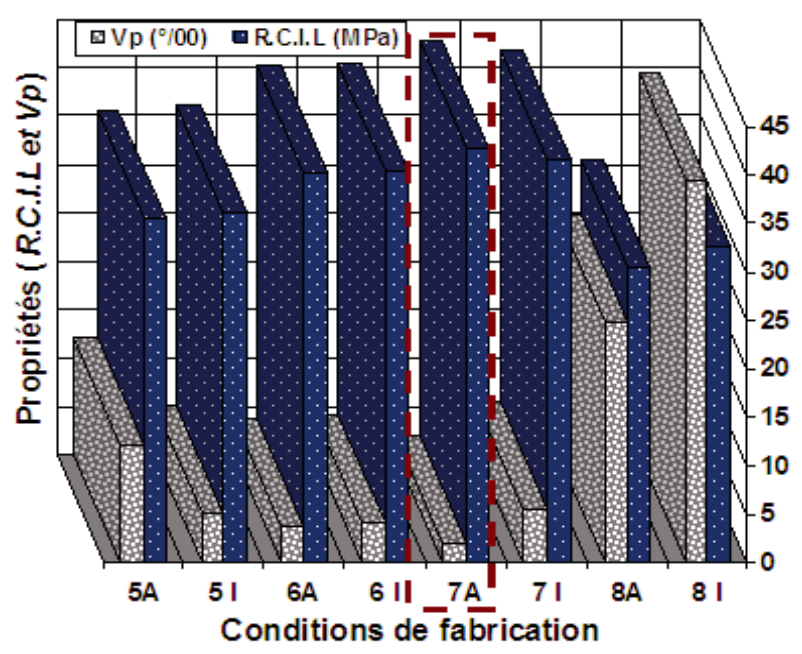

Fig. 15. Comparaison entre conditions de fabrication du plan d'expérience.

Fig. 15. Comparison of manufacturing process conditions of design of experiments.

(LRI : liquid resin infusion) ont été identifiés. Ils sont a priori le nombre de couches de carbone NCF constituant la préforme, le nombre de couches de tissu drainant, les températures de moule, d'injection et de cuisson de résine époxy RTM 6, le côté aspiration de vide ou injection de résine où se situe la pièce structurelle dans le moule pendant le processus de fabrication. Ces paramètres ont permis de concevoir un plan d'expérience selon plusieurs conditions de fabrication. L'interaction entre le nombre de couches de carbone NCF et le nombre de couches de tissu drainant a aussi de l'influence sur les propriétés telles que la résistance au cisaillement interlaminaire RCIL et la fraction volumique en porosités $V_{\mathrm{p}}$ des composites infusés.

L'analyse statistique a montré que le nombre de couches de NCF, les températures de moule et de cuisson de résine et l'interaction entre le nombre de couches de NCF et de drainant affectent la fraction volumique en porosités des composites infusés. En fonction des conditions de fabrication, les observations au microscope électronique à balayage (MEB) présentent des tailles et des formes de porosités différentes. Ce qui explique et confirme les résultats des réponses en cisaillement interlaminaire trouvés. La valeur de la RCIL est fortement influencée par le taux de porosités que contient le matériau. Son degré de qualité atteint affecte considérablement cette caractéristique. Les conditions optimales qui permettent d'obtenir les meilleures propriétés dans le cadre de cette étude sont l'utilisation d'un seul tissu drainant associé à des basses températures de fabrication mesurées sur la face supérieure de la plaque.

La suite de cette étude sera consacrée à l'étude des relations existant entre ces paramètres de fabrication et d'autres propriétés de la structure composite infusée [28] (température de transition vitreuse, fraction volumique en fibres, résistance en traction et compression...) et entre les différentes propriétés d'autre part en utilisant un plan d'expérience plus complet. Dans un second temps, l'objectif est d'étudier les effets du renforcement dans l'épaisseur par couture des préformes sur les propriétés mécaniques et la santé matière des structures stratifiées infusées.

\section{Références}

[1] S.W. Beckwith, Sampe J. 42 (2006) 1

[2] SAERTEX, SAERTEX GmbH\&Co, 2005, Datashett(KG), 2

[3] HEXFLOW, http://www.hexcel.com/NR/ rdonlyres/B9DAF85C-DFA5-4158-8CE522A8F7146A94/0/HEXCELRTM6.pdf, online, editor

[4] F. Berthet, Contribution à l'étude de l'influence des paramètres de mise en œuvre et de constitution sur les propriétés mécaniques des composites carbone/époxy injectés sur renfort (RTM), in Sciences et Génie des Matériaux, soutenu le 4 mars 1998, Thèse de doctorat, École des Mines de Paris, 326

[5] J.R. Thagard, O.I. Okoli, Z. Liang, J. Reinforced Plast. Comp. 23 (2004) 12

[6] L.R. Thomas, A.K. Miller, A.L. Chan, Fabrication of complex high-performance composite structures at low cost using VARTM, 47th International SAMPE Symposium 47 (2002) 13 
[7] B. Yenilmez, M. Senan, E.M. Sozer, Comp. Sci. Tech. (2008) 28

[8] K. Dransfield, C. Baillie, Y.-W. Mai, Comp. Sci. Tech. 50 (1994) 305-317

[9] Q. Govignon et al., Composites Part A : Appl. Sci. Manufacturing 39 (2008) 1412-1426

[10] Han, et al., Composites Part A : Appl. Sci. Manufacturing (Incorporating Composites and Composites Manufacturing) 34 (2003) 10

[11] F. Zhou, J. Alms, S.G. Advani, Comp. Sci. Tech. 68 (2007) 699-708

[12] L. Teemer, O.I. Okoli, Z. Liang, SAMPE '06 Long Beach April 51 (2006) 9

[13] P. Krawczak, Essais des plastiques renforcés, in Plastiques et Composites, Techniques de l'Ingénieur, Doc. AM 5405 (2008) 36

[14] S.C. Amouroux, Sampe J. 42 (2006) 10

[15] C.-L. Lee, K.-H. Wei, J. Appl. Polymer Sci. 77 (1999) 7

[16] N.R.L. Pearce, Composites : Part A (29A) (1998) 19-27

[17] J.S. Leclerc, E. Ruiz, Composites : Part A 39 (2008) 1859-1868

[18] L. Liu et al., Composite Structures 73 (2006) 303-309

[19] E. Ruiz et al., Comp. Sci. Tech. 66 (2006) 475486

[20] C. Dong, Chensong, Comp. Sci. Tech. 68 (2008) 2125-2133
[21] A. Lamure, Méthodologie des plans d'expériences : plans factoriels complets, in Cours INP : ENSIACET, 2007

[22] R. Sabre, Procédés chimie-bio-agro, Apport du génie des procédés, 2007, Doc. F 1 006, 10

[23] M. Labrosse, Techniques de l'Ingénieur. Plastiques et Composites (Doc. A 3 523) (1998) 35

[24] University of Delaware and Center for Composite Materials, VARTM Variability and Substantiation. The Joint Advanced Materials and Structures Center of Excellence, May 2005, 32

[25] Y. Ledru et al., The 9th International Conference on Flow Processes in Composite Materials, Montréal (Québec), Canada, 2008, FPCM-9, 9

[26] E. Ruiz, F. Trochu, Composites Part A : Appl. Sci. Manufacturing 36 (2005) 806-826

[27] E. Ruiz, F. Trochu, Polymer Composites 26 (2005) 209-230

[28] A. Njionhou, F. Berthet, B. Castanie, Parameters affecting mechanical properties of composite manufactured by liquid resin infusion (LRI) in ICCM 17 17th International Conference on Composite Materials, 2009, Edinburgh, UK 